\title{
FM1-43 Imaging Reveals cGMP-Dependent Long-Term Depression of Presynaptic Transmitter Release
}

\author{
Patric K. Stanton, ${ }^{1}$ Uwe Heinemann, ${ }^{2}$ and Wolfgang Müller ${ }^{2}$ \\ ${ }_{1}^{1}$ Departments of Neuroscience and Neurology, Albert Einstein College of Medicine, Bronx, New York 10461-1602, and \\ 2Neuroscience Research Institute, Charité, Humboldt University, Berlin, D-10117, Germany
}

\begin{abstract}
A persistent question concerning mechanisms underlying longterm, activity-dependent synaptic plasticity is whether the sites of alterations are presynaptic, postsynaptic, or both. Recently, we discovered a chemical method of inducing long-term depression (LTD) of synaptic strength at Schaffer collateral-CA1 synapses by simultaneously elevating [CGMP] and inhibiting cAMP-dependent protein kinase (PKA). Chemical LTD (CLTD) is activity-independent, occluded by stimulus-evoked LTD, and requires access of pharmacologic agents to presynaptic terminals. In the present study, we used fluorescence and twophoton imaging of presynaptic terminals with the fluorescent dye $\quad \mathrm{N}$-(3-triethylammoniumpropyl)-4-(4-(dibutylamino)styryl) pyridinium dibromide (FM1-43) to determine directly if inducing CLTD is associated with a long-term reduction in transmitter release. In presynaptic Schaffer collateral-CA1 terminals of control hippocampal slices loaded with FM1-43, electrical stimulation (10 Hz/2 min) elicited a frequency-dependent destaining
\end{abstract}

Long-term depression (LTD) is a persistent decrease in synaptic strength in response to prolonged low-frequency synaptic stimulation. In conjunction with long-term potentiation (LTP), LTD has been suggested to play important roles in memory storage processes. LTP and LTD of synaptic strength might result from alterations on both presynaptic and postsynaptic sides of the synapse. Changes in presynaptic transmitter release are one such change (Bekkers and Stevens, 1990; Malinow and Tsien, 1990; Bolshakov and Siegelbaum, 1994). Postsynaptic changes that have been associated with LTP and LTD include phosphorylation of glutamate receptors (Barria et al., 1997; Nicoll and Malenka, 1999; Lee et al., 2000), unmasking of new receptors at silent synapses (Liao et al., 1995), and modifications of dendritic spine shape and electrotonic properties (Muller et al., 2000).

Betz and Bewick (1992) have used activity-dependent uptake of fluorescent molecules to directly monitor presynaptic vesicle dynamics. The most commonly used fluorescent molecules for this purpose are members of the styryl dye family, including $N$-(3-triethylammoniumpropyl)-4-(4-(dibutylamino)styryl) pyridinium dibromide (FM1-43), that exhibit prominent fluorescence

\footnotetext{
Received March 23, 2001; revised July 3, 2001; accepted June 15, 2001.

This work was supported by Whitehall Foundation and Alexander von Humboldt Foundation Grants to P.K.S. and Deutsche Forschungsgemeinschaft and Charité Grants to W.M. This work is dedicated to the memory of Lewis N. Stanton, Sr. We thank Eric Kandel, John Connor, Christopher Bailey, and Russell Nichols for helpful discussions, and Henning Glasser and Karin Berlin for expert technical assistance.

Correspondence should be addressed to Dr. Patric K. Stanton, Department of Neuroscience, Albert Einstein College of Medicine, 1300 Morris Park Avenue, Bronx, NY 10461-1602. E-mail: stanton@aecom.yu.edu.

Copyright (C) 2001 Society for Neuroscience 0270-6474/01/210001- $\$ 15.00 / 0$
}

that peaked at $20 \%$ reduction in fluorescence. In contrast, when we first induced CLTD by a 30 min treatment of slices with the type $\mathrm{V}$ phosphodiesterase inhibitor zaprinast $(20 \mu \mathrm{M})$ plus the PKA inhibitor $N$-[2-( $p$-bromocinnamylamino)ethyl]-5isoquinolinesulfonamide $(\mathrm{H}-89 ; 10 \mu \mathrm{M})$, then washed for $60 \mathrm{~min}$, the destaining of FM1-43 fluorescence evoked by the same stimulation was reduced to $4 \%$. Treatment and washout of slices with either drug singly had a significantly smaller effect on stimulus-evoked FM1-43 destaining. Only CLTD was associated with virtually complete suppression of stimulus-evoked FM1-43 release, the first direct evidence for at least one form of LTD being mediated by persistent reduction of presynaptic transmitter release.

Key words: CA1; cGMP; hippocampus; long-term depression; presynaptic terminal; Schaffer collateral; PKA; PKG; transmitter release

after insertion of their hydrophobic tail into lipid bilayers. FM1-43 has yielded important insights into endocytosis, exocytosis, and vesicular release by single synaptic terminals between cultured neurons and at the neuromuscular junction (Betz and Bewick 1992; Ryan et al., 1993, Betz et al., 1996; Cochilla et al., 1999).

The acute brain slice has become a favored preparation for the study of synaptic physiology because of the combination of in vitro stability while minimizing alterations from in vivo neuronal architecture. Recently, Pyle et al. (1999), in an elegant approach, overcame the problem of excessive background fluorescence by membrane-bound FM1-43 in brain slices. They applied the aqueous fluorophore sulforhodamine-101 (S-Rhd) to transfer the energy of adjacent FM1-43 emission in the extracellular space to a longer wavelength by fluorescence resonance energy transfer (FRET), whereas the rapid decay of FRET with distance from S-Rhd molecules leaves vesicle bound FM1-43 unaffected. By

This article is published in The Journal of Neuroscience, Rapid Communications Section, which publishes brief, peerreviewed papers online, not in print. Rapid Communications are posted online approximately one month earlier than they would appear if printed. They are listed in the Table of Contents of the next open issue of JNeurosci. Cite this article as: JNeurosci, 2001, 0:RC167 (1-6). The publication date is the date of posting online at www.jneurosci.org.

http://www.jneurosci.org/cgi/content/full/5625 
using a bandpass filter to exclude S-Rhd fluorescence, they successfully resolved vesicular fluorescence.

We used FM1-43 and the method of Pyle et al. (1999) to image presynaptic release in control slices and in slices in which LTD of synaptic strength had been induced. Because vesicles loaded first with FM1-43 lose dye when LTD is induced by electrical stimulation and depolarizing to load dye after induction of LTD could modify or erase it (Fleck et al., 1992), we exploited our recent discovery of a purely chemical, activity-independent form of LTD (Santschi et al., 1999) to study its effects on transmitter release. Chemical LTD (CLTD) is induced by raising intracellular [cGMP] with the type $\mathrm{V}$ phosphodiesterase inhibitor zaprinast, while simultaneously inhibiting PKA with $N$-[2-( $p$-bromocinnamylamino)ethyl]-5-isoquinolinesulfonamide (H-89). While our previous studies suggest that these compounds induce LTD by actions on presynaptic terminals (Reyes-Harde et al., 1999; Santschi et al., 1999), direct evidence of a change in transmitter release has been lacking. Therefore, we used fluorescence and twophoton laser scan microscopy to determine directly whether CLTD is associated with long-lasting reductions in stimulusevoked release of FM1-43 from Schaffer collateral-CA1 terminals.

\section{MATERIALS AND METHODS}

Hippocampal slice preparation. Wistar rats, 15-21 d of age, were decapitated under deep ether anesthesia, their brains removed, and hippocampi dissected free in ice-cold artificial CSF (ACSF) (in mM): $\mathrm{NaCl} \mathrm{126;} \mathrm{KCl}$ 5; $\mathrm{NaH}_{2} \mathrm{PO}_{4}$ 1.25; $\mathrm{MgCl} 2 ; \mathrm{CaCl}_{2} 2 ; \mathrm{NaHCO}_{3} 26$; and glucose 10. A hippocampal block from the middle third was glued to a Teflon base with cyanoacrylate adhesive, submerged in ice-cold oxygenated ACSF $(95 \%$ $\mathrm{O}_{2}$ and $5 \% \mathrm{CO}_{2}$ ), and $350-\mu \mathrm{m}$-thick slices were cut in the transverse axis with a Camden Vibroslice (WPI, Sarasota, FL). Slices were immediately placed in an interface holding chamber and incubated at $25^{\circ} \mathrm{C}$ for at least $1 \mathrm{hr}$ before transfer to a submerged recording chamber on the microscope stage, also at $25^{\circ} \mathrm{C}$. Slices were perfused with ACSF (2 $\mathrm{ml} / \mathrm{min}$ ) saturated with $95 \% \mathrm{O}_{2}$ and $5 \% \mathrm{CO}_{2}$, and drugs were bathapplied in the same ACSF.

FM1-43 staining and destaining in slices. After confirming the presence of Schaffer collateral-evoked EPSPs $>1 \mathrm{mV}$ in amplitude in CA1 stratum radiatum, $10 \mu \mathrm{M}$ 6-cyano-7-nitroquinoxaline-2,3-dione (CNQX) was bath-applied throughout to prevent synaptically driven action potentials in CA3 pyramidal neurons from accelerating dye release. Synaptic boutons were loaded by bath-applying $8 \mu \mathrm{M}$ FM1-43 (Molecular Probes, Eugene, OR) in $40 \mathrm{~mm}\left[\mathrm{~K}^{+}\right]$ACSF for $15 \mathrm{~min}$, then returning to normal ACSF. Individual boutons were imaged after $30 \mathrm{~min}$ perfusion with dye-free ACSF plus $50 \mu \mathrm{M}$ sulforhodamine-101 (Molecular Probes). Stimulus-induced destaining by bipolar stimulation ( $150 \mu \mathrm{sec}$ DC pulses) with a $20 \mathrm{~Hz} / 2 \mathrm{~min}$ train, or 5,20 , or 50 pulse bursts given each $15 \mathrm{sec}$ (see Fig. $2 B$ ). Depolarization-dependent destaining was evoked by bathapplying $80 \mathrm{~mm}\left[\mathrm{~K}^{+}\right]$ACSF.

Fluorescence microscopy. Fluorescent images were acquired by a cooled digital, back-illuminated CCD camera (Princeton Instruments, Trenton, NJ), using a Zeiss $40 \times 0.75$ numerical aperture water immersion objective on a Zeiss Axioskop microscope. FM1-43 was excited with $480 \pm 6 \mathrm{~nm}$ (Polychrome IV Monochromator; T.I.L.L. Photonics, Grafelfing, Germany) for $20 \mathrm{msec}$ signal integration, and emission light was filtered at $535 \pm 20 \mathrm{~nm}$ (Omega Optical, Brattleboro, VT). Images were digitized using the ST138 Detector Controller (Princeton Instruments), displayed and analyzed using custom-made software. Rectangular regions of interest (ROIs; $\sim 2-4 \mu \mathrm{m}^{2}$ ) were defined around the center of bright, punctate fluorescence spots, and 9-12 boutons and 3-4 background fields were measured in each slice. If lateral displacement of a bouton beyond the ROI occurred, the data set was discarded. A fluorescence time course was generated by normalizing each ROI time course by dividing by starting intensity, averaging the background fields to produce a dye-bleaching time course ( $2 \mathrm{hr}$ bleaching, $12.7 \pm 1.1 \%$ ), and then dividing each bouton ROI by bleaching at corresponding time points throughout the experiment. Vertical bars denote SEM for the average of all normalized and corrected boutons across experiments.

Two-photon microscopy. We visualized FM1-43 fluorescence with two-

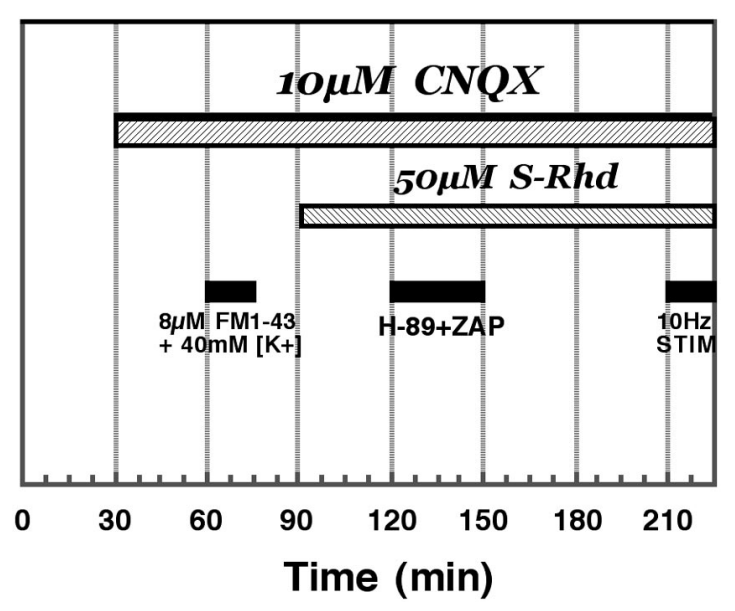

Figure 1. Time course of drug applications in the CLTD protocol. After confirming slice viability with Schaffer collateral-evoked EPSPs, $10 \mu \mathrm{M}$ CNQX was bath-applied to block synaptic excitation. Thirty minutes later, presynaptic terminals were loaded by $15 \mathrm{~min}$ application of $8 \mu \mathrm{M}$ FM1-43 in $40 \mathrm{mM}\left[\mathrm{K}^{+}\right]$( first solid bar), nonspecific fluorescence was quenched with $50 \mu \mathrm{M}$ S-Rhd, and then CLTD was induced by application of $20 \mu \mathrm{M}$ zaprinast plus $10 \mu \mathrm{M} \mathrm{H}-89(H-89+Z A P)$ for $30 \mathrm{~min}$. One hour after washout of H-89 plus ZAP, FM1-43 destaining was evoked by application of $10 \mathrm{~Hz}$ Schaffer collateral stimulation.

photon excitation, using a Leica (Nussloch, Germany) DM LFS E upright microscope with water-immersion objective APO L $63 \times / 0.90 \mathrm{~W}$ and a Leica multispectral confocal laser scan unit. The light source was a Millenia $5 \mathrm{~W}$ diode laser source pumping a Tsunami Ti:sapphire laser (Spectra-Physics, Mountain View, CA) that provided $\approx 130 \mathrm{fsec}$ pulses at $82 \mathrm{MHz}$, tuned to $840 \mathrm{~nm}$ center wavelength. Epifluorescence was detected with photomultiplier tubes of the confocal laser scan head with pinhole maximally opened and emission spectral selection optimized for signal over background $(560-660 \mathrm{~nm})$. A higher wavelength range supplied brighter emission levels for two-photon imaging in the absence of S-Rhd, relative to fluorescence emission bandpass $(535 \pm 20 \mathrm{~nm})$ in S-Rhd. Laser intensity was controlled with a variable beam splitter exploiting polarization of the laser light and neutral density filters. Though there were no signs of photodamage, we always used the lowest intensity necessary for adequate signal-to-noise ratio.

\section{RESULTS}

\section{Quenching of background fluorescence in FM1-43- stained hippocampal slices}

The aqueous S-Rhd is used extensively as a fluorescent conjugate to molecules of biological interest and as an acceptor for fluorescent resonance energy transfer (Stryer, 1978). Recently, Pyle et al. (1999) used S-Rhd co-bath-applied with FM1-43 to absorb unwanted nonvesicular FM1-43 fluorescence. Paralleling their method, we used an excitation wavelength of $480 \pm 6 \mathrm{~nm}$ and an emission filter with a narrow bandpass at $535 \pm 20 \mathrm{~nm}$, a range of wavelengths over which FM1-43 emits but S-Rhd does not.

Figure 1 shows our experimental protocol. After determination that Schaffer collateral-evoked population EPSPs $>1 \mathrm{mV}$ in amplitude, exhibiting paired-pulse facilitation (50 msec interval), could be recorded in CA1 stratum radiatum, $10 \mu \mathrm{M}$ CNQX was applied for the remainder of experiments to prevent synaptically driven CA3 action potentials from eliciting FM1-43 release. Thirty minutes later, $8 \mu \mathrm{M}$ FM1-43 plus $40 \mathrm{~mm}\left[\mathrm{~K}^{+}\right]_{\mathrm{o}}$ was applied for $15 \mathrm{~min}$ to load presynaptic terminals, then $\left[\mathrm{K}^{+}\right]_{\mathrm{o}}$ was returned to $5 \mathrm{~mm}$. CLTD was induced only after FM1-43 loading to avoid the possibility that $40 \mathrm{~mm}\left[\mathrm{~K}^{+}\right]_{\mathrm{o}}$ might reverse plastic changes in release properties. Thereafter, addition of micromolar concentrations of S-Rhd dramatically attenuated $(>80 \%)$ fluorescence 

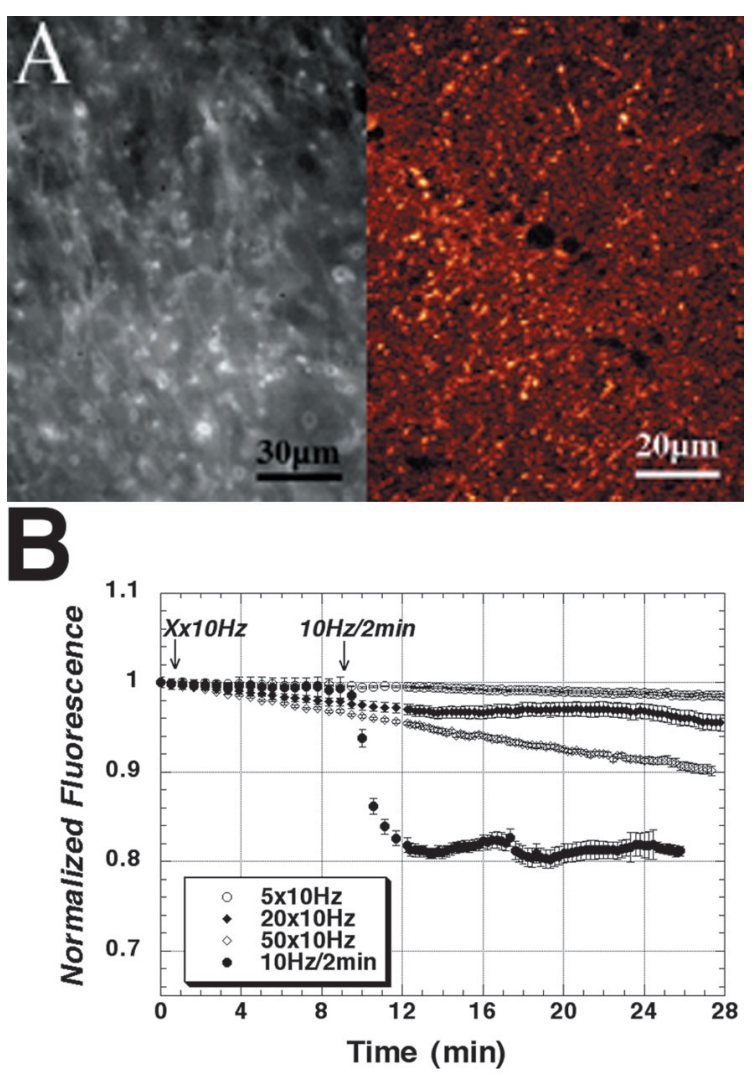

Figure 2. Stimulus dependence of FM1-43 destaining in CA1 stratum radiatum of hippocampal slices. A, Typical fluorescence (left; excitation $480 \pm 12 \mathrm{~nm}$, emission $535 \pm 20 \mathrm{~nm}$ filtering) and two-photon (right; 800 $\mathrm{nm}$ excitation, $>600 \mathrm{~nm}$ emission filtering) images of presynaptic puncta labeled with FM1-43. B, Time course of stimulus-induced reductions in FM1-43 fluorescence evoked by repeated $10 \mathrm{~Hz}$ bursts of 5 (open circles), 20 ( filled diamonds), or 50 (open squares) stimuli applied each $15 \mathrm{sec}$, or a continuous 2 min train of $10 \mathrm{~Hz}$ stimuli (filled circles). Each point is mean \pm SEM of $17-24$ presynaptic puncta from three or four slices.

from superficial membrane-bound FM1-43, causing emergence of bright, punctate fluorescent spots representing FM1-43-loaded presynaptic terminals not accessible to S-Rhd (Fig. 2A, left). Drugs were applied for $30 \mathrm{~min}$, then washed for an additional 60 min, before synaptic stimulation was given to assess long-term effects on the efficacy of transmitter release.

\section{Duration dependence of stimulus-evoked FM1-43 destaining}

First, we examined the effects of a range of stimulus durations on FM1-43 destaining in different groups of slices using standard fluorescence imaging. In all plots, fluorescence for each puncta was divided by starting fluorescence to normalize to one, then each time point was divided by the normalized fluorescence of background fields at that time point. In this way, all time courses were corrected for loss of fluorescence caused by bleaching of FM1-43, yielding stable baseline fluorescence time courses. Figure $2 B$ illustrates mean time courses for four different stimulus protocols in which $10 \mathrm{~Hz}$ Schaffer collateral stimulation was applied each $15 \mathrm{sec}$ in repeated bursts of 5, 20, or 50 stimuli or in a continuous $10 \mathrm{~Hz} / 2 \mathrm{~min}$ train. Stimulating each $15 \mathrm{sec}$ elicited approximately linear destaining time courses that were more rapid when the number of stimuli in each burst was increased, whereas the continuous 2 min train of stimuli elicited a rapid and marked drop in FM1-43 fluorescence that averaged a $20 \%$ reduc- tion across all 36 puncta from four slices. At the end of each experiment, application of $80 \mathrm{~mm}\left[\mathrm{~K}^{+}\right]_{\mathrm{o}}$ elicited an additional drop in FM1-43 fluorescence to $60 \%$ of initial values within 2 min.

To directly confirm that FM1-43 destaining we observed was attributable to stimulus-evoked presynaptic, rather than postsynaptic, release, we examined destaining in slices in which postsynaptic neurons were silenced. Previous studies (Alici et al., 1996) have shown that prolonged (90-120 min) application of glucosefree ACSF to hippocampal slices produces severe damage to CA1 pyramidal neurons that completely eliminates the ability to sustain postsynaptic resting membrane or synaptic potentials, whereas presynaptic stimulus-evoked ionic fluxes and vesicular release recover relatively completely. After $90 \mathrm{~min}$ in glucose-free ACSF, hippocampal slices were returned to normal ACSF, loaded with FM1-43, and stimulus-evoked FM1-43 destaining was examined. Stimulus-evoked FM1-43 destaining in slices subjected to glucose-free ACSF was indistinguishable from control slices, supporting the conclusion that FM1-43 destaining is primarily attributable to presynaptic vesicular release mechanisms.

\section{Induction of CLTD by simultaneously raising [cGMP] and inhibiting PKA produces LTD of presynaptic transmitter release}

In previous electrophysiological studies (Santschi et al., 1999), we showed that elevating [cGMP] while simultaneously inhibiting PKA elicits CLTD of synaptic strength at Schaffer collateralCA1 synapses. In this study, [cGMP] was increased by zaprinast, a potent and selective inhibitor of type $\mathrm{V}$ phosphodiesterase, the enzyme that selectively degrades cGMP, while PKA was inhibited with the synthetic inhibitor $\mathrm{H}-89$.

Slices were first loaded with FM1-43 in $40 \mathrm{~mm}\left[\mathrm{~K}^{+}\right]_{\mathrm{o}}$ for 15 min, washed for $30 \mathrm{~min}$, then zaprinast plus H-89 was bathapplied for $30 \mathrm{~min}$ to induced CLTD and washed for $60 \mathrm{~min}$ before commencing imaging. Figure $3 A$ summarizes this imaging in untreated, control slices (open circles), versus slices in which CLTD had been induced ( filled circles). In control slices $(n=24$ puncta from four slices), Schaffer collateral stimulation $(10 \mathrm{~Hz} / 2$ min) (Fig. $3 A$, arrow) produced a rapid, selective $20 \%$ reduction in fluorescence of FM1-43-labeled puncta, consistent with stimulus-dependent release of vesicularly stored FM1-43 from presynaptic boutons. In contrast, slices in which CLTD had been induced ( $n=24$ puncta from four slices) exhibited markedly less stimulus-evoked FM1-43 destaining ( $p<0.05$; Student's $t$ test compared with control puncta), consistent with our hypothesis that CLTD is associated with a long-term reduction in glutamate release. As a final control for the specificity of FM1-43 fluorescent puncta, we applied $80 \mathrm{~mm}\left[\mathrm{~K}^{+}\right]_{\mathrm{o}}$ at the end of each experiment (solid bars). An $80 \mathrm{~mm}$ concentration of $\left[\mathrm{K}^{+}\right]_{\mathrm{o}}$, which should cause complete release of vesicularly sequestered FM1-43, elicited a further reduction in FM1-43 fluorescence in both control and CLTD slices, down to the same floor of $\sim 60 \%$ of original, prestimulus baseline.

\section{Two-photon images of FM1-43-stained presynaptic boutons confirm that CLTD is associated with LTD of presynaptic transmitter release}

To more clearly image presynaptic FM1-43 fluorescent boutons and to confirm their punctate nature and selective destaining with stimulation, we used two-photon excitation to confocally excite FM1-43 fluorescence. The right image of Figure $2 A$ illustrates a typical two-photon image of FM1-43-labeled puncta in CA1 stratum radiatum. The use of two-photon imaging offered three distinct advantages over conventional fluorescence imaging: (1) 

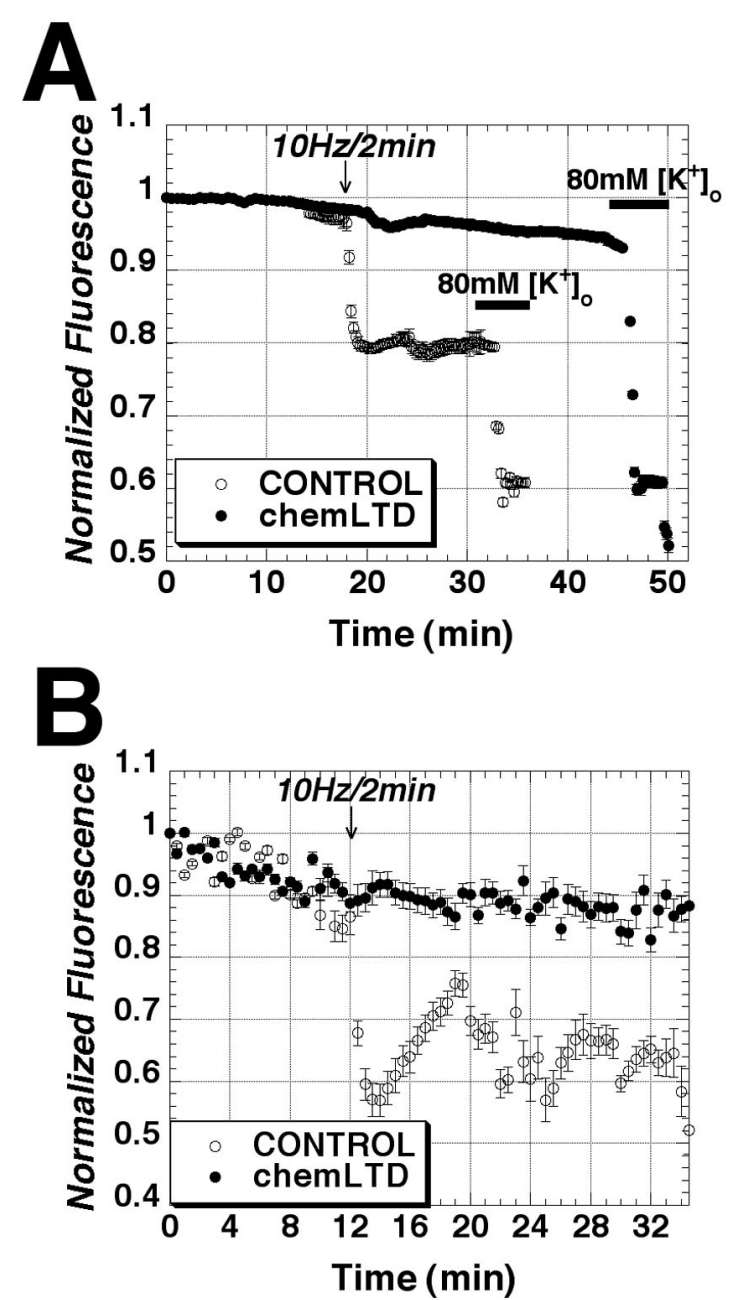

Figure 3. CLTD persistently reduces stimulus-induced release of FM143. A, Time course of Schaffer collateral-evoked $(10 \mathrm{~Hz} / 2 \mathrm{~min}$; arrow $)$ FM1-43 destaining from conventional fluorescence images in stratum radiatum of field CA1 in control slices (open circles) versus those in which CLTD had been induced $1 \mathrm{hr}$ before stimulation (chemLTD; filled circles). At the end of each experiment, $80 \mathrm{~mm}\left[\mathrm{~K}^{+}\right]$(solid bars) was bath-applied to completely release the remaining vesicularly stored FM1-43. Each point is mean \pm SEM of 24 presynaptic puncta from four slices. $B$, Time course of two-photon excitation measurements of FM1-43 destaining in field CA1 elicited by Schaffer collateral stimulation $(10 \mathrm{~Hz} / 2 \mathrm{~min}$; arrow $)$ in control slices (open circles) versus slices in which CLTD had been induced $1 \mathrm{hr}$ before start of imaging (chemLTD; filled circles). Each point is mean \pm SEM of 13-17 presynaptic puncta from three slices.

superior resolution of presynaptic boutons, (2) focal excitation eliminated the need for sulforhodamine-101 to quench nonvesicular fluorescence, and (3) bleaching was significantly reduced by the highly focal nature of the excitation. We used the same experimental protocol as in Figure 1 to determine whether the induction of CLTD is associated with similar reductions in stimulus-evoked FM1-43 destaining of two-photon puncta. Figure $3 B$ is a plot of the time course of these experiments, in control versus CLTD slices. In control slices (open circles; $n=13$ synaptic puncta from three slices), Schaffer collateral stimulation $(10 \mathrm{~Hz} / 2$ min) (Fig. 3B, arrow) elicited marked decreases in FM1-43 fluorescence of two-photon imaged puncta, larger in magnitude than conventional fluorescence imaging experiments. In contrast, slices where CLTD had been induced (filled circles; $n=17$ synaptic puncta from three slices) showed significantly less
FM1-43 destaining when the same stimulus was given $(p<0.05$; Student's $t$ test compared with control puncta). These experiments confirm, with a different technique, that the induction of CLTD with zaprinast plus $\mathrm{H}-89$ is associated with a reduction in presynaptic transmitter release at Schaffer collateral-CA1 synaptic terminals.

\section{Effect of transient elevation of [cGMP] alone on FM1-43 destaining}

To test directly whether elevating intracellular [cGMP] alone elicits LTD of transmitter release, we transiently applied zaprinast $(20 \mu \mathrm{M})$ to slices previously loaded with FM1-43. After 30 min zaprinast bath application and $60 \mathrm{~min}$ drug washout, we began imaging FM1-43-loaded presynaptic puncta. Figure $4 A$ illustrates the time course of these experiments, starting $60 \mathrm{~min}$ after wash. Interestingly, FM1-43 fluorescence slowly declined throughout the prestimulus period, consistent with continuing release of FM1-43 from presynaptic terminals. However, when synaptic stimulation was applied $(10 \mathrm{~Hz} / 2 \mathrm{~min})$ (Fig. $4 A$, arrow), a rapid, stimulus-evoked FM1-43 destaining was observed in stratum radiatum ( $n=36$ puncta from three slices). Although smaller than controls (12 vs $20 \%$ ), stimulus-evoked destaining was significantly larger than during CLTD $(p<0.05$; one-way ANOVA compared with CLTD and control) and reached final destained levels similar to control slices.

\section{Effect of transient inhibition of PKA alone on FM1-43 destaining}

To test whether inhibiting PKA by itself has long-term effects on stimulus-evoked FM1-43 destaining at Schaffer collateral-CA1 synapses, we bath-applied the selective PKA inhibitor H-89 (10 $\mu \mathrm{M})$ to slices loaded with FM1-43. After a 30 min drug application and 60 min washout, we began imaging FM1-43-loaded puncta. Figure $4 B$ illustrates the time course of these experiments, starting $60 \mathrm{~min}$ after wash. Unlike zaprinast, H-89 did not have long-term effects on baseline leakage of FM1-43 from presynaptic terminals, evidenced by the stable baseline before electrical stimulation. However, Schaffer collateral stimulation $(10 \mathrm{~Hz} / 2 \mathrm{~min})$ (Fig. 4B, arrow) evoked a rapid, stimulus-evoked FM1-43 destaining of $12 \%$ in stratum radiatum $(n=36$ puncta from three slices; $p<0.05$, one-way ANOVA compared with CLTD and control).

\section{DISCUSSION}

For years, retrograde messengers have been hypothesized to mediate presynaptic changes triggered by postsynaptic induction of synaptic plasticity. However, Casado et al. (2000) reported evidence for postsynaptic effects of forward diffusion of nitric oxide triggered by presynaptic NMDA receptors. We sought to directly test whether presynaptic reductions in vesicular release can accompany LTD of synaptic strength.

CLTD is based on signal transduction pathways necessary for stimulus-evoked LTD (Gage and Stanton, 1997; Reyes-Harde et al., 1999). The ability of stimulus-evoked LTD to occlude expression of CLTD suggests that the two phenomena share at least some transduction mechanisms (Santschi et al., 1999). Retrograde activation of guanylyl cyclase by nitric oxide and presynaptic involvement of cGMP suggest a presynaptic site of expression for at least some forms of LTD, a conclusion confirmed by our current evidence for reduced vesicular release during CLTD.

Pyle et al. (1999) first successfully used FM1-43 to image vesicular release in slices, by using sulforhodamine-101 to quench background fluorescence. Consistent with their findings, we found sulforhodamine-101 to be extremely effective in absorbing 
A

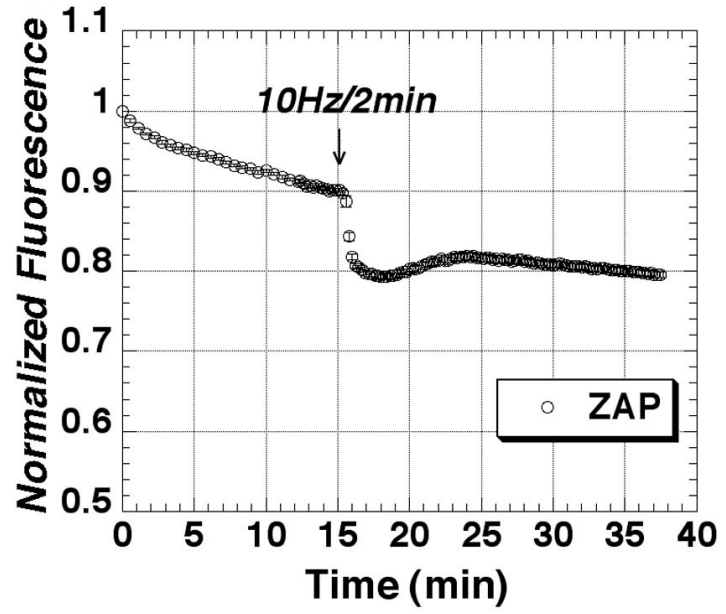

B

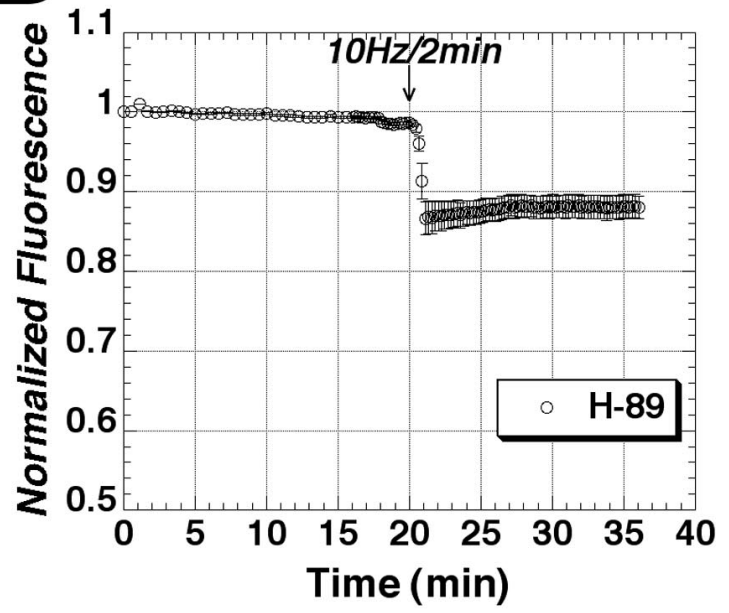

Figure 4. Zaprinast or H-89 alone do not persistently reduce stimulusevoked release of FM1-43. A, Time course of Schaffer collateral-evoked (10 Hz/2 min; arrow) FM1-43 destaining in stratum radiatum of field CA1 of slices pretreated for 30 min with $10 \mu \mathrm{M} \mathrm{H}-89$, then washed for $1 \mathrm{hr}$ before start of imaging. Each point is mean \pm SEM of 12 presynaptic puncta from three slices. $B$, Time course of Schaffer collateral-evoked (10 $\mathrm{Hz} / 2$ min; arrow) FM1-43 destaining in stratum radiatum of field CA1 of slices pretreated for $30 \mathrm{~min}$ with $20 \mu \mathrm{M}$ zaprinast, then washed for $1 \mathrm{hr}$ before start of imaging. Each point is mean \pm SEM of 12 presynaptic puncta from three slices.

fluorescence from surface membrane-bound FM1-43; fluorescent puncta that remained showed behavior completely consistent with presynaptic boutons releasing vesicularly sequestered FM143. In presence of CNQX to minimize spontaneous synaptically driven dye release, fluorescent puncta still showed a slow decline ( $12.7 \%$ over $2 \mathrm{hr}$ ), perhaps because of spontaneous dye release and/or bleaching, which was corrected by normalizing fluorescence time courses to kinetics of nonpunctate, background decay (consistent with bleaching). After this correction, fluorescent puncta intensities were stable, and exhibited rapid, stimulusevoked destaining consistent with release of FM1-43 into the extracellular space, where fluorescence was diluted and absorbed by S-Rhd. Moreover, evoked destaining showed clear dependence on stimulus number; short $10 \mathrm{~Hz}$ bursts evoked much less destaining than a continuous 2 min train.
Coapplication of zaprinast, which inhibits type $\mathrm{V}$ phosphodiesterase and raises [cGMP], plus the PKA inhibitor H-89, markedly and persistently reduced stimulus-evoked FM1-43 release, consistent with a presynaptic site of expression of CLTD. The fact that both conventional fluorescence in S-Rhd and twophoton imaging without S-Rhd yielded similar results strengthens this conclusion. Furthermore, application of either drug alone, conditions that do not evoke CLTD (Santschi et al., 1999), had a much smaller effect on stimulus-evoked FM1-43 release. In these earlier studies, we confirmed near-complete washout of each drug individually well within $60 \mathrm{~min}$. In contrast to the typical magnitude of CLTD of synaptic transmission (40-50\%; Santschi et al., 1999), CLTD of FM1-43 release exhibited up to a $75 \%$ decrease. This could suggest that FM1-43 labels a pool of vesicles that preferentially take up dye and that these are more prone to expression of LTD, and perhaps other forms of activitydependent plasticity as well.

There was an intriguing difference in effects of zaprinast and H-89. Whereas H-89 application and wash yielded stable baseline FM1-43 fluorescent puncta, zaprinast was associated with a substantial continuing decay in fluorescence during post-drug washout (Fig. 4A). Because data are already normalized for dye bleaching, we suggest that zaprinast reduces spontaneous transmitter release, as it does evoked (Boulton et al., 1994), and that this caused a rebound enhancement in spontaneous basal release after drug washout. It is also notable that there was no such decay when zaprinast was coapplied with $\mathrm{H}-89$, suggesting that the rebound increase in spontaneous release is PKA-dependent.

Although both fluorescence and two-photon imaging yielded similar conclusions, there were striking differences. The restricted energy profile of two-photon excitation eliminated the need for $\mathrm{S}$-Rhd to absorb background fluorescence. We observed more movement artifacts during and immediately after stimulation with this method, consistent with stimulus-induced cellular swelling. Although movement may have contributed to greater variability in two-photon experiments, shifts, when they occurred, recovered within 2-4 min after stimulation.

A question of continuing debate is whether presynaptic or postsynaptic alterations underlie LTP and LTD. Answers to this question have been confounded by assumptions that the site is one or the other, that stimulus-evoked LTP and LTD are unitary phenomena, and that methods of measuring presynaptic transmitter release are largely indirect and open to alternative interpretations. A purely chemical method of inducing LTD through altering cyclic nucleotide second messengers offered the prospect of being a more restricted phenomenon. Our previous work (Santschi et al., 1999) supplied indirect evidence that CLTD is expressed at presynaptic loci. This FM1-43 study supports the hypothesis that CLTD is expressed, at least partially, by a persistent, cGMP-dependent, reduction in presynaptic transmitter release.

The purely chemical nature of the CLTD paradigm is useful at inducing CLTD throughout a slice after loading with FM1-43. We are currently working to improve our two-photon imaging sensitivity to test directly for long-term reductions in release from single boutons after induction of activity-dependent LTD, especially since studies have indicated a presynaptic locus for some forms of stimulus-evoked LTD (Bolshakov and Siegelbaum, 1994; Stanton and Gage, 1996; Gage and Stanton, 1997; Reyes-Harde et al., 1999),

Increases in [cGMP] have been implicated in induction of both LTP (Arancio et al., 2001) and LTD (Gage et al., 1997; Reyes- 
Harde et al., 1999), with the level of PKA activation appearing to be a critical switch for expression of LTP versus LTD. Our data support an important role for presynaptic, in addition to postsynaptic, PKA in long-term synaptic plasticity. One action of presynaptic cGMP required for LTD appears to be stimulation of presynaptic ryanodine receptor-mediated release of $\mathrm{Ca}^{2+}$ (Reyes and Stanton, 1996; Reyes-Harde et al., 1999). The question of how presynaptic $\mathrm{Ca}^{2+}$ is involved in a signal transduction chain resulting, under conditions of reduced PKA activity, in long-term reductions in release probability, remains to be elucidated.

\section{REFERENCES}

Alici K, Müller W, Heinemann U (1996) Effects of glutamate receptor agonists on presumed presynaptic $\mathrm{Ca}(2+)$-signals in juvenile rat hippocampal area CA1. Neurosci Lett 214:187-190.

Arancio O, Antonova I, Gambaryan S, Lohmann SM, Wood JS, Lawrence DS, Hawkins RD (2001) Presynaptic role of cGMP-dependent protein kinase during long-lasting potentiation. J Neurosci 21:143-149.

Barria A, Muller D, Derkach V, Griffith LC, Soderling TR (1997) Regulatory phosphorylation of AMPA-type glutamate receptors by CaMKII during long-term potentiation. Science 276:2042-2045.

Bekkers JM, Stevens CF (1990) Presynaptic mechanism for long-term potentiation in the hippocampus. Nature 346:724-729.

Betz WJ, Bewick GS (1992) Optical analysis of synaptic vesicle recycling at the frog neuromuscular junction. Science 255:200-203.

Betz WJ, Mao F, Smith CB (1996) Imaging exocytosis and endocytosis. Curr Opin Neurobiol 6:365-371.

Bolshakov VY, Siegelbaum SA (1994) Postsynaptic induction and presynaptic expression of hippocampal long-term depression. Science 264:1148-1152.

Boulton CL, Irving AJ, Southam E, Potier B, Garthwaite J, Collingridge GL (1994) The nitric oxide-cyclic GMP pathway and synaptic depression in rat hippocampal slices. Eur J Neurosci 6:1528-1535.

Casado M, Dieudonne S, Ascher P (2000). Presynaptic N-methyl-Daspartate receptors at the parallel fiber-Purkinje cell synapse. Proc Natl Acad Sci USA 97:11593-11597.
Cochilla AJ, Angelson JK, Betz WJ (1999) Monitoring secretory membrane with FM1-43 fluorescence. Annu Rev Neurosci 22:1-10.

Fleck MW, Palmer AM, Barrionuevo G (1992) Potassium-induced longterm potentiation in rat hippocampal slices. Brain Res 580:100-105.

Gage AT, Stanton PK (1997) Nitric oxide-guanylyl-cyclase-dependent and independent components of multiple forms of long-term synaptic depression. Hippocampus 7:286-295.

Lee HK, Barbarosie M, Kameyama K, Bear MF, Huganir RL (2000) Regulation of distinct AMPA receptor phosphorylation sites during bidirectional synaptic plasticity. Nature 405:955-959.

Liao D, Hessler NA, Malinow R (1995) Activation of postsynaptically silent synapses during pairing-induced LTP in CA1 region of hippocampal slice. Nature 375:400-404.

Malinow R, Tsien RW (1990) Presynaptic enhancement shown by whole-cell recordings of long-term potentiation in hippocampal slices. Nature 346:177-180.

Muller D, Toni N, Buchs PA (2000) Spine changes associated with longterm potentiation. Hippocampus 10:596-604.

Nicoll RA, Malenka RC (1999) Expression mechanisms underlying NMDA receptor-dependent long-term potentiation. Ann NY Acad Sci 868:515-525.

Pyle JL, Kavalali ET, Choi S, Tsien RW (1999) Visualization of synaptic activity in hippocampal slices with FM1-43 enabled by fluorescence quenching. Neuron 24:803-808.

Reyes M, Stanton PK (1996) Induction of hippocampal long-term depression requires release of $\mathrm{Ca}^{2+}$ from separate presynaptic and postsynaptic intracellular stores. J Neurosci 16:5951-5960.

Reyes-Harde M, Empson R, Potter BVL, Galione A, Stanton PK (1999) Evidence of a role for cyclic ADP-ribose in long-term synaptic depression in hippocampus. Proc Natl Acad Sci USA 96:4061-4066.

Ryan TA, Reuter H, Wendland B, Schweizer FE, Tsien RW, Smith SJ (1993) The kinetics of synaptic vesicle recycling measured at single presynaptic boutons. Neuron 11:713-724.

Santschi LA, Reyes-Harde M, Stanton PK (1999) Chemically-induced, activity-independent LTD elicited by simultaneous activation of PKG and inhibition of PKA. J Neurophysiol 82:1577-1589.

Stanton PK, Gage AT (1996) Distinct synaptic loci of $\mathrm{Ca}^{2+} /$ calmodulindependent protein kinase II necessary for long-term potentiation and depression. J Neurophysiol 76:2097-2101.

Stryer L (1978) Fluorescence energy transfer as a spectroscopic ruler. Ânnu Rev Biochem 47:819-846. 